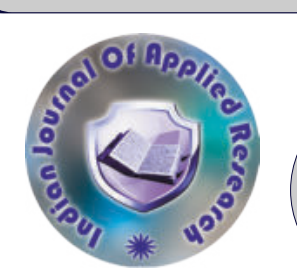

Radio diagnosis

\title{
COMPARISON OF SEMI-AUTOMATED CT VOLUMETRY AND INTRA- OPERATIVE GRAFT WEIGHT IN POTENTIAL DONORS FOR LIVING DONOR LIVER TRANSPLANTATION (LDLT)
}

Srikanth Moorthy

\section{G. Shibu}

\section{Sanjana} Devarmani*
Department of Radiodiagnosis, Amrita Institute of Medical Sciences, Amrita Viswa Vidyapeetham, Cochin, Kerala, India.

Department of Radiodiagnosis, Amrita Institute of Medical Sciences, Amrita Viswa Vidyapeetham, Cochin, Kerala, India.

Department of Radiodiagnosis, Amrita Institute of Medical Sciences, Amrita Viswa Vidyapeetham, Cochin, Kerala, India. ${ }^{*}$ Corresponding Author

\section{ABSTRACT AI}

AIM: The aim is to study the correlation between estimated graft weight (EGW) and actual graft weight (AGW) in potential donors for LDLT. MATERIALS AND METHODS: The study cohort consisted of 133 right lobe (RL) with middle hepatic vein (MHV), 23 RL without MHV and 17 left lobe (LL) grafts assessed between August 2018 and August 2020. EGW was calculated using semi-automated volumetry technique in Philips IntelliSpace portal workstation and AGW was measured intra-operatively by the transplant team after graft retrieval from the donor. To find the agreement between AGW and EGW, intra-class correlation co-efficient (ICC) was computed. To find the degree of correlation between EGW and AGW, Spearman's rho correlation co-efficient was computed. Bland Altman plot was used for assessing the level of agreement between two methods. RESULTS: The mean age of donors was $38.9+/-9.9$ years (Female:Male::121:52). The correlation between EGW and AGW for all three types of grafts was significant ( $\mathrm{p}<0.001)$. RL with MHV: Spearman's rho r=0.786, ICC $=0.883$; RL without MHV: Spearman's rho $r=0.968$, ICC $=0.986$ and LL: Spearman's rho $r=0.809$, ICC $=0.937$. Bland Altman's analysis showed that CT volumetry overestimated 5 and underestimated 1 RL grafts with MHV, overestimated 1 RL grafts without MHV and underestimated 1 LL grafts. CONCLUSION: CT volumetry has a definitive role in the pre-operative evaluation of prospective liver donors. EGW calculated by semi-automated CT volumetry in potential LDLT donors is accurate and correlates well with AGW measured intra-operatively for both right and left lobe grafts.

\section{KEYWORDS : Liver, Computed tomography, Liver transplantation}

\section{INTRODUCTION}

Liver transplantation (LT) is the treatment of choice for end-stage liver disease (ESLD) caused by a wide spectrum of etiologies like alcoholic cirrhosis or viral hepatitis. It is an important curative option for patients with acute and chronic liver failure and hepatocellular carcinoma (HCC) who have not responded to other treatments. Due to the rampant increase in the demand for donor livers outstripping the donor availability, LDLT has become a viable alternative to deceased donor liver transplantation (DDLT). Studies of LDLT have shown acceptable results in terms of short-term survival and graft outcomes compared with DDLT with full size organ and long-term donor quality of life (1, 2). A liver remnant, about $30-40 \%$ of the original liver volume is required for the donor to survive. A minimum of $40 \%$ of the standard liver mass, which is calculated from body surface area or graft recipient weight ratio (GRWR) of $>/=0.8$, is needed by the recipient (3). Therefore, an accurate liver volumetry technique is necessary in the pre-transplant work up of donors for planning a liver transplant. CT may be a good tool for this purpose as it is easily available, noninvasive, a faster imaging modality and has high spatial and contrast resolution $(4,5)$. The objective of this study was to assess the correlation between EGW calculated by CT volumetry and AGW measured intra-operatively.

\section{MATERIALS AND METHODS}

Institutional Review Board approval was taken for this prospective study. Informed consent was taken from all the patients before they underwent computed tomography (CT). We included 173 liver donors who underwent pre-transplant work up in our institution between August 2018 to August 2020. All the prospective donors willing to donate liver for transplantation were included in the study. Patients excluded from the study were, patients with severe fatty liver, focal hepatic lesions, insufficient liver volumetry, complex vascular anatomy and serology positive for $\mathrm{HIV}, \mathrm{HBsAg}$ and $\mathrm{HCV}$.

All liver donors underwent a detailed pre-operative evaluation according to our institutional protocol. It included multiphase CT abdomen with contrast for vascular anatomy and volumetry and magnetic resonance cholangiopancreatography for biliary anatomy. Imaging was performed using 256 slice MDCT (BrillianceiCT;Philips Healthcare, Cleveland, $\mathrm{OH}$ ) after intravenous injection of non-ionic iodinated contrast agent, Iohexol (Omnipaque ${ }^{\mathrm{TM}} 350$, GE Healthcare, Princeton, NJ) over a period of 25 seconds. A $1.5 \mathrm{~mL} / \mathrm{Kg}$ body weight of the contrast was administered to the patients. The contrast was injected using power injector (OptiVantage, Guerbet, $\mathrm{OH}$ ). Bolus triggering was used to obtain the contrast enhanced phases. The region of interest was placed over descending thoracic aorta, $2 \mathrm{~cm}$ proximal to the diaphragm and scanning was initiated after the threshold of $100 \mathrm{HU}$ was reached. Arterial dominant phase images were acquired at 15 seconds, portal dominant phase images were acquired at 45 seconds and delayed hepatic venous phase images were acquired at 100 seconds after the injection.

CT volumetry was performed using semi-automated volumetry technique in Philips Intellispace Portal Workstation using axial delayed venous phase images of $5 \mathrm{~mm}$ reconstruction thickness. The CT volumetry information was used to calculate the estimated graft volume (Fig 1). A conversion factor of 1 was used to convert CT estimated graft volumes in cubic $\mathrm{cm} /$ milliliters to grams of liver, which is the estimated graft weight. After retriveal of the liver graft, AGW was measured on back table in the operation theatre. After which the correlation between $\mathrm{AGW}$ and $\mathrm{EGW}$ was computed.

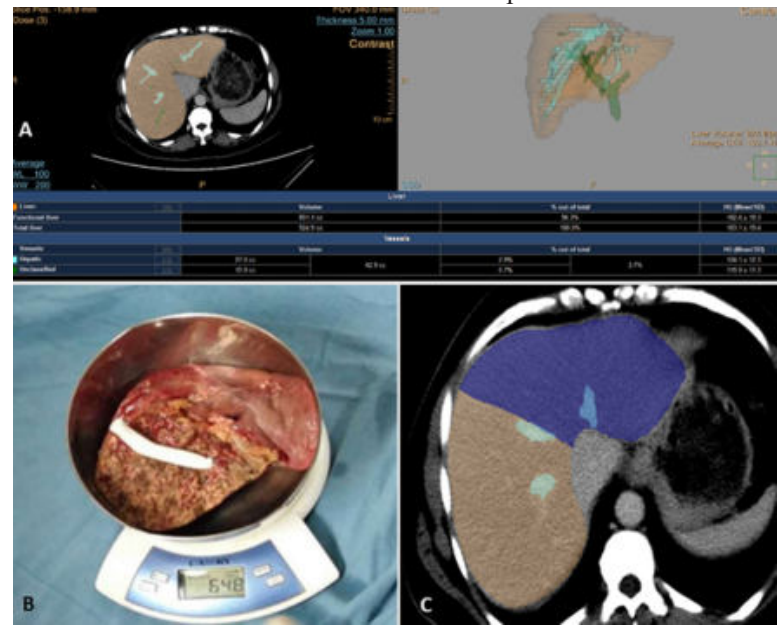

Fig 1: A 36-year old female prospective liver donor who underwent pre-transplant CT Volumetry. A) Semiautomated volumetry technique in 256 slice CT Philips Intellispace Portal workstation. B) Intraoperative measurement of graft weight. C) Segmental liver volume. 


\section{STATISTICALANALYSIS:}

Statistical analysis was done using IBM SPSS 20. (SPSS Inc, Chicago, USA). To find the agreement between actual graft weight (AGW) and estimated graft weight (EGW) for liver weights, intra-class correlation co-efficient was computed. To find the degree of correlation between EGW and AGW, Spearman's rho correlation co-efficient was computed. Bland Altman plot created by plotting the difference between EGW and AGW against mean of EGW and AGW and the $95 \%$ confidence interval (mean $\pm 1.96 \times \mathrm{SD}$ ) was used to assess over and underestimation of graft volumes by CT volumetry in comparison to intra-operative graft weights.

\section{RESULTS}

The mean age of donors was $38.9+/-9.9$ years (Female: Male:: 121:52). We studied 133 (76.9\%) right lobe (RL) grafts with MHV, 23 (13.3\%) RL grafts without MHV and 17 (9.8\%) left lobe (LL) grafts. The correlation between EGW and AGW for all three types of grafts was significant $(\mathrm{p}<0.001)$. RL with MHV: Spearman's rho $\mathrm{r}=0.786$, ICC $=0.883$; RL without MHV: Spearman's rho $\mathrm{r}=0.968, \mathrm{ICC}=0.986$ and LL: Spearman's rho $\mathrm{r}=0.809$, ICC $=0.937$ (Table 1). Bland Altman's analysis showed that CT volumetry overestimated 5 and underestimated $1 \mathrm{RL}$ grafts with MHV, overestimated $1 \mathrm{RL}$ graft without MHV and underestimated 1 LL grafts (Table 2).

Table 1: Intraclass correlation and Spearman's rho correlation between EGW and AGW

\begin{tabular}{|l|l|l|}
\hline & $\begin{array}{l}\text { Intra class } \\
\text { correlation }\end{array}$ & $\begin{array}{l}\text { Spearman's rho } \\
\text { correlation }\end{array}$ \\
\hline Right lobe grafts with MHV & 0.883 & $0.786, \mathrm{p}<0.001$ \\
\hline Right lobe grafts without MHV & 0.986 & $0.968, \mathrm{p}<0.001$ \\
\hline Left lobe grafts & 0.937 & $0.809, \mathrm{p}<0.001$ \\
\hline
\end{tabular}

Table 2: Bland Altman analysis for right and left lobe grafts

\begin{tabular}{|l|l|}
\hline & Bland Altman analysis \\
\hline Right lobe grafts with MHV & $\begin{array}{l}\text { Mean: }+98 \mathrm{~g} \text { [Range: }-88 \mathrm{~g} \text { to } \\
+284 \mathrm{~g}] \\
\text { Overestimation: } 5 \\
\text { Underestimation: } 1\end{array}$ \\
\hline Right lobe grafts without MHV & $\begin{array}{l}\text { Mean: }+67 \mathrm{~g} \text { [Range: }-12 \mathrm{~g} \text { to } \\
+146 \mathrm{~g}] \\
\text { Overestimation: } 1\end{array}$ \\
\hline Left lobe grafts & $\begin{array}{l}\text { Mean: }-17.6 \mathrm{~g} \text { [Range: }-70.5 \mathrm{~g} \text { to } \\
+105.8 \mathrm{~g}] \\
\text { Underestimation: } 1\end{array}$ \\
\hline
\end{tabular}

\section{DISCUSSION}

Liver transplantation is the treatment of choice for end-stage liver disease (ESLD). The healthy donor is subjected to a hemihepatectomy, a surgical procedure with significant risks and without apparent medical benefits to the donor. Donor selection is based on total and segmental liver volumes, because assuring appropriate graft size is one of the major predictors of a safe, successful outcome for both donor and recipient. A graft that is too small has increased likelihood of dysfunction secondary to inadequate functional hepatic mass and possible excessive portal perfusion. A large graft is associated with a risk of graft compression and poor perfusion. In addition, it should be made sure that an adequate volume of liver is retained in potential donors to permit metabolic functions during the regeneration process. Therefore, accuracy of total and segmental liver volumes is important to avoid donor-recipient volume mismatch. Hence, an accurate noninvasive volumetry technique is quintessential for planning a liver transplant.

In the present study ( $\mathrm{n}=173), 121(69.9 \%)$ were females and 52 $(30.1 \%)$ were males. The liver donors were mostly young and middleaged adults, the mean age of the study population being $38.99 \pm 9.91$ years. Out of 173 grafts in the present study, 133 (76.9\%) were RL grafts with MHV, $23(13.3 \%)$ were RL grafts without MHV and 17 $(9.8 \%)$ were left lobe (LL) grafts. In a similar study conducted by Radtke et al, they obtained 49 RL grafts with MHV, 06 RL grafts without MHV and 07 LL grafts (6). In this study, we obtained a statistically significant strong correlation between EGW and AGW for RL grafts with MHV (Spearman's correlation $r=0.786$ and $p<0.001$ ), RL grafts without MHV (Spearman's correlation $\mathrm{r}=0.968$ and $\mathrm{p}<$ 0.001 ) and LL grafts (Spearman's correlation $r=0.809$ and $p<0.001$ ). This is consistent with a study conducted by Sanjay Goja et al in 842 donors, in which they found a statistically significant strong correlation between EGW and AGW for RL grafts $(\mathrm{r}=0.82, \mathrm{P}<0.001)$ and LL grafts $(\mathrm{r}=0.81, \mathrm{P}<0.001)$ and statistically significant moderate correlation between EGW and AGW for LLS grafts $(\mathrm{r}=0.49, \mathrm{P}<0.001)$ (7). A study by T. Yoneyama et al also showed that the correlation coefficient between EGW and AGW was 0.84 in right lobe grafts and 0.85 in left lobe grafts $(\mathrm{p}<0.001)$, indicating a statistically significant correlation which is consistent with our results (8). In our study, we also found that there is statistically good agreement between EGW and AGW for RL grafts with MHV (ICC $=0.883$ ), and statistically excellent agreement between EGW and AGW RL grafts without MHV $(\mathrm{ICC}=0.986)$ and LL grafts $(\mathrm{ICC}=0.937)$. Bland Altman's analysis showed that $\mathrm{CT}$ volumetry overestimated 5 and underestimated $1 \mathrm{RL}$ grafts with MHV, overestimated 1 RL grafts without MHV and underestimated 1 LL grafts. In a similar study by Sanjay Goja et al $(\mathrm{N}=842)$, they found that the EGW was both over and under estimated in RL grafts, EGW was overestimated in LL grafts and was underestimated in LLS grafts (7).

\section{CONCLUSIONS:}

The correlation between EGW (calculated in preoperative CT) and AGW (measured intra-operatively) was statistically significant for both right and left lobe liver grafts. Hence, CT volumetry has a definitive role in the preoperative evaluation of prospective liver donors and donor selection.

\section{REFERENCES:}

1. Hoehn RS, Wilson GC, Wima K, Hohmann SF, Midura EF, Woodle ES, et al. Comparing living donor and deceased donor liver transplantation: A matched national analysis from 2007 to 2012: National Analysis of Living Liver Donor Liver Transplantation. Liver Transpl. 2014 Nov;20(11):1347-55.

2. Bhatti ABH, Zia H, Dar FS, Zia MQ, Nasir A, Saif F, et al. Quality of Life After Living Donor Hepatectomy for Liver Transplantation. World J Surg. 2015 Sep;39(9):2300-5.

3. Suzuki K, Epstein ML, Kohlbrenner R, Garg S, Hori M, Oto A, et al. Quantitative Radiology: Automated CT Liver Volumetry Compared With Interactive Volumetry and Manual Volumetry. Am J Roentgenol. 2011 Oct 1;197(4):W706-12.

4. Nakayama $\mathrm{Y}, \mathrm{Li} Q$, Katsuragawa S, et al. Automated hepatic volumetry for living related liver transplantation at multisection CT. Radiology 2006;240:743-8

5. Kamel IR, Kruskal JB, Keogan MT, Goldberg SN, Warmbrand G, Raptopoulos V. Multidetector CT of Potential Right-Lobe Liver Donors. Am J Roentgenol. 2001 Sep 1;177(3):645-51.

6. Radtke A, Sotiropoulos GC, Nadalin S, Molmenti EP, Schroeder T, Saner FH, et al Preoperative volume prediction in adult live donor liver transplantation: 3-D CT Volumetry approach to prevent miscalculations. Eur J Med Res. 2008;8.

7. Goja S, Yadav SK, Yadav A, Piplani T, Rastogi A, Bhangui P, et al. Accuracy of preoperative CT liver volumetry in living donor hepatectomy and its clinical preoperative CT liver volumetry in living donor hepatect

8. Yoneyama T, Asonuma K, Okajima H, Lee K-J, Yamamoto H, Takeichi T, et al. Coefficient factor for graft weight estimation from preoperative computed tomography volumetry in living donor liver transplantation. Liver Transpl. 2011;17(4):369-7. 